\title{
Surface Chemistry of Active Carbon: Specific Adsorption of Phenols
}

\author{
JAMES S. MATTSON, ${ }^{1}$ HARRY B. MARK, JR., ${ }^{1}$ MICHAEL D. MALBIN, ${ }^{1}$ \\ WALTER J. WEBER, JR., ${ }^{2}$ AND JOHN C. CRITTENDEN ${ }^{2}$ \\ Departments of Chemistry ${ }^{1}$ and ${ }^{2}$ Civil Engineering, University of Michigan, \\ Ann Arbor, Michigan 48104 \\ Received: February 24, 1969
}

\begin{abstract}
The characteristics of the uptake of phenol and nitrophenols by active carbon have been investigated. The measurements of solution equilibrium parameters as well as surface structural characteristics determined by infrared internal reflection spectroscopy are presented. A charge-transfer interaction is postulated which explains the observed adsorption and spectroscopic characteristics on the basis of the electron densities in the sorbate molecule, as well as the types and concentrations of surface functional groups present on the carbon.
\end{abstract}

\section{INTRODUCTION}

The mechanisms by which active carbon functions to remove phenols from aqueous solutions have never been clearly defined. A thorough understanding of these sorptive mechanisms is essential for accomplishing the most effective use of active carbon in water and waste water treatment.

Several previous research efforts have recognized the importance of developing information regarding sorptive phenomena associated with active carbon in aqueous solutions of phenols (1-4). Although different experimental approaches have been employed, all of these studies involved the interpretation of surface properties from measurement of changes in bulk solution parameters. Indirect interpretations of this type often are only speculative with regard to possible surface interaction mechanisms. The present study of the adsorption of phenol and nitrophenols makes use of a recently developed application of infrared internal reflection (IRS) spectroscopy (5). This technique permits direct spectrophotometric observation of solid surfaces, and therefore provides a direct means for the identification of the organic functional group characteristics at the surface, both before and after adsorption.

There has been a considerable amount of indirect study on the nature of the oxygencontaining functional groups present on the surfaces of carbons $(2,5-12)$. It has been observed that oxidation and reduction of active carbon markedly influences the nature of the phenol and nitrobenzene isotherms (2). Coughlin and Ezra (2) showed that oxidation of active carbon considerably lowers the capacity of both active carbon and carbon black for adsorption of phenoland nitrobenzene in the low solute concentration regions of the isotherms. They also observed that reduction of the carbon resulted in the opposite effect. It was suggested (2) that adsorption of phenol takes place with the pielectron system of the graphatic rings of the carbon basal planes (4), and that the presence of additional acidic surface oxygen groups produced by oxidation of the carbon, at the basal plane edges, serves to withdraw electrons from the pi-system of the basal

Journal of Colloid and Interface Science, Vol. 31, No. 1, September 1969 
planes (2). This does not explain (2) how such an electron withdrawal effect makes itself felt over the large distances of the basal ring system, nor does it explain the nature of interaction of the sorbates on the basal planes.

Giles et al. (4) pointed out that the adsorption isotherm for phenol on carbon usually shows a two-step process, resulting in two plateaus. The oxidation-reduction studies of Coughlin and Ezra (2) showed that this second plateau was apparently independent of the oxidation state of the carbon surface. Giles (4) suggested that the second step of the isotherm involved an uncovering of part of the surface and readsorption of the phenol molecules in a different orientation. It was suggested (4) that this reorientation involved a change from a flat configuration to an end-on configuration where the hydroxyl group is directed away from the carbon surface.

From consideration of previous speculation, it was evident that considerably more information was necessary with respect to the nature of the adsorption of phenols before the actual mechanism could be resolved. Certain of this necessary information was obtained from the study of adsorption isotherms of phenol, the meta- and para-isomers of nitrophenol, and nitrobenzene on active carbon in the low concentration range of the isotherms presented in this paper. This characteristic adsorption data, in conjunction with infrared internal reflectance spectra of $p$-nitrophenol in the adsorbed state on active carbon are employed as the basis for the postulation of a general charge-transfer mechanism theory for this type of interaction.

\section{MATERIALS AND METHODS}

Adsorption Isotherm Studies. A ligninbased carbon was used ${ }^{3}$ in these studies which

${ }^{3}$ Nuchar C-1000, obtained from the West Virginia Pulp and Paper Co., Carbon Technical Center, Covington, Va., Sample $67 \times 244$.

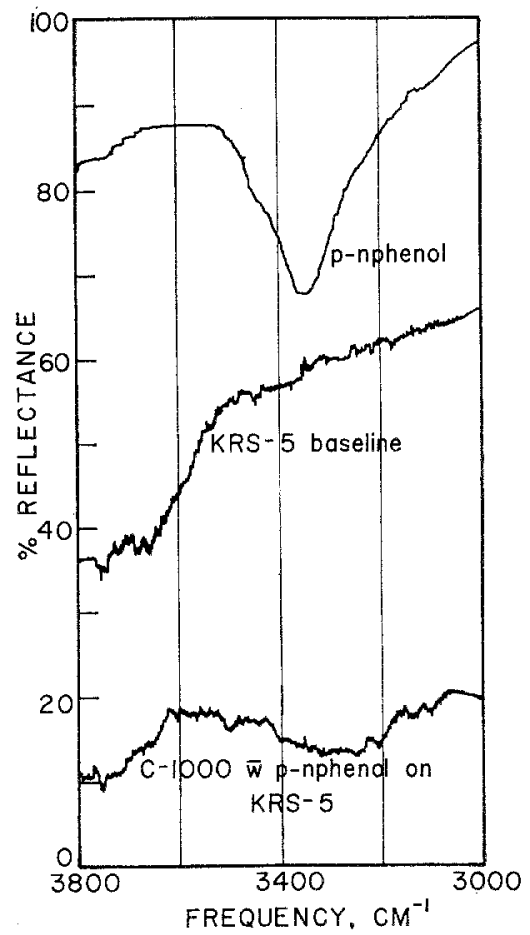

FIG. 1. O-H stretching region. The top speetrum shows the $\mathrm{O}-\mathrm{H}$ band of solid $p$-nitrophenol (KRS-5, $45^{\circ}$ ). The lower spectrum is that of active carbon with $p$-nitrophenol adsorbed on the carbon

$\left.\mathrm{K} \quad \mathrm{S}-5,60^{\circ}, 10 \times\right)$.

had a $\mathrm{N}_{2}$-BET surface area of about 1000 $\mathrm{m}^{2} / \mathrm{gm} .^{4}$ Before use the carbon was first washed in triply distilled water to remove water soluble ash, dried, and then sieved through a 325 -mesh screen $(44 \mu)$ to obtain a distribution of small particle sizes. The carbon was again washed in triply distilled water to remove fines, then dried. Drying this material was accomplished by heating to $200^{\circ} \mathrm{C}$ for $12-24$ hours and allowing it to cool in a vacuum desiccator. This drying procedure was deemed necessary because both the infrared spectra and the measurement of oxygen content (5) previously had indicated that such carbons contained up to about $8 \%$ water after drying at $105^{\circ} \mathrm{C}$. (The bottom infrared spectrum in Fig. 1 shows that the

${ }^{4}$ Charles R. Nichols, West Virginia Pulp and Paper Co., Covington Va., personal communication. 
$3.0-\mu \mathrm{O}-\mathrm{H}$ peak observed in earlier work (5) disappeared when the carbon was dried as described above). The samples of carbon were stored under vacuum in a desiccator until used. The $p$-nitrophenol employed ${ }^{3}$ was recrystallized four times from toluene, and had a $113-114^{\circ} \mathrm{C}$ melting point. The $m$-nitrophenol ${ }^{3}$ was decolorized with active carbon in hot toluene and subsequently recrystallized twice from toluene. The purified $m$-nitrophenol had a melting point range of $97-99^{\circ} \mathrm{C}$. Nitrobenzene ${ }^{3}$ and phenol ${ }^{4}$ were employed without further purification.

The adsorption isotherms were obtained using $100 \mathrm{ml}$ of solution and from 15 to 150 $\mathrm{mg}$ of active carbon per sample. A separate sample was used for each point on the isotherm, and the $\mathrm{pH}$ of the solutions was adjusted to maintain a constant value. Samples were continually agitated in bottles with polyethylene-lined caps by rotating them end-for-end in a tumbler. The time required for the equilibration of phenol samples was approximately $20 \mathrm{~min}$, while the other solutes equilibrated in less than $1 \mathrm{~min}$. However, all phenol samples were allowed 24 hours for equilibration, and the other samples at least $20 \mathrm{~min}$. Separation of the carbon from the solutions was done by filtration through microporous filters. ${ }^{5}$ Solute concentrations were then measured with either a Cary Model 14 or a Beckman DU spectrophotometer. $^{6}$ Desorption experiments were performed on $p$-nitrophenol samples by filtering all of the equilibrated solution from an isotherm sample, followed by quantitative transfer of the carbon to a fresh solution of triply distilled water made up to the proper

\footnotetext{
${ }^{3}$ Matheson, Coleman and Bell white Iabel (best grade).

${ }^{4}$ Mallinckrodt Analytical Reagent grade.

5 Millipore GSWP $0.22 \mu$ filters.

- Phenol was determined colorimetrically at either $2105 \AA\left(\epsilon=6,200 \mathrm{e}-\mathrm{mole}^{-1}-\mathrm{cm}^{-1}\right)$ or $2700 \AA$ $(\epsilon=1540)$; nitrobenzene at $2685 \AA(\epsilon=11,900)$; $p$-nitrophenol at $3170 \AA(\epsilon=9,800)$; and $m$-nitrophenol at $2720 \AA(\epsilon=5808)$. The weak acids were colorimetrically determined in $\mathrm{pH} 2$ solution (HC1).
}

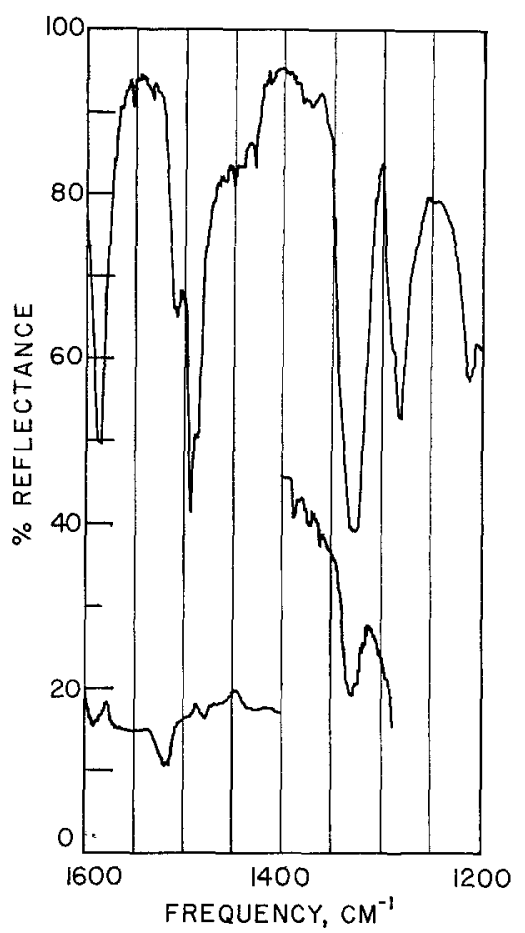

Fig. 2. 1600-1200 $\mathrm{Cm}^{-1}$ region. The top spectrum shows the $\mathrm{NO}_{2}$ group bands and $\mathrm{C}-\mathrm{O}$ band of $p$-nitrophenol. The lower spectrum shows the same bands of $p$-nitrophenol adsorbed on active carbon.

$\mathrm{pH}$ with $\mathrm{HCl}$. The cycle was repeated as often as desired (up to 20 times in the studies reported here). The equilibration time was $20 \mathrm{~min}$. All temperatures were $25^{\circ} \mathrm{C}$ unless otherwise stated.

Relative rates of adsorption were determined with 5 liters of solution, at room temperature, constantly stirring at a constant rate of $1000 \mathrm{rpm}$. A total of $50 \mathrm{ml}$ of solution was withdrawn in 5-ml aliquots, at appropriate time intervals, and the carbon separated by filtration through microporous filters supported in a syringe filter holder. ${ }^{7}$.

Characterization of Carbon Structure. Photomicrograph slides were prepared by suspending the carbon in a solution of $20 \%$ collodion-butylacetate and by placing a few

${ }^{7}$ Gelman Instrument Co., Ann Arbor, Mich., Syringe filter holder no. 4320 and GA-6 $0.45 \mu$ filters. 
drops of this solution on a large water surface. A microscope slide was then brought up through the film. After oven drying, the preparation on the slide is quite stable.

Internal Reftection Spectra. The internal reflectance spectrum of pure $p$-nitrophenol was obtained with a thin film (ca. $400 \AA$ ) of the solid $p$-nitrophenol by evaporating an acetone solution on a KRS-5, $45^{\circ}$, IRS crystal. By employing this thin film method, there are no significant depth-of-penetration effects, and the spectrum closely approximates a transmission spectrum (14). All IRS spectra were obtained using a pair of Wilks Scientific Corp. MIR-9 IRS accessories in the sample compartment of a Perkin-Elmer Model 621 spectrophotometer. The cell compartment was continually flushed with dry, $\mathrm{CO}_{2}$-free air to eliminate atmospheric interferences resulting from slightly different path lengths for the sample and reference beams. For the internal reflection spectral studies of the carbon samples, both KRS-5 and Ge IRs crystals were employed. It has been found that it is not feasible to determine the spectra of carbon with adsorbed aromatic compounds using germanium IRS crystals because of the tendency of the adsorbate to migrate to the germanium surface which results in readsorption on the germanium (5). Thus, for these studies, KRS-5 was used as the internal reflection element (12).

It should be noted that the carbon/ germanium critical angle is about $27-31^{\circ}$, while the KRS-5/carbon critical angle is a minimum of $59^{\circ}$ at $2.5 \mu$. At $7.51 \mu$, the carbon has a refractive index greater than the KRS-5 $\left(n_{\mathrm{c}}=2.42(15) ; n_{\mathrm{KRS}-5}=2.4\right)$ and it is not, therefore, possible to obtain total internal reflection under these conditions. Thus, the lower right hand spectrum in Fig. 2 , where the phenolic $\mathrm{O}-\mathrm{C}$ band of adsorbed $p$-nitrophenol is observed using a KRS-5

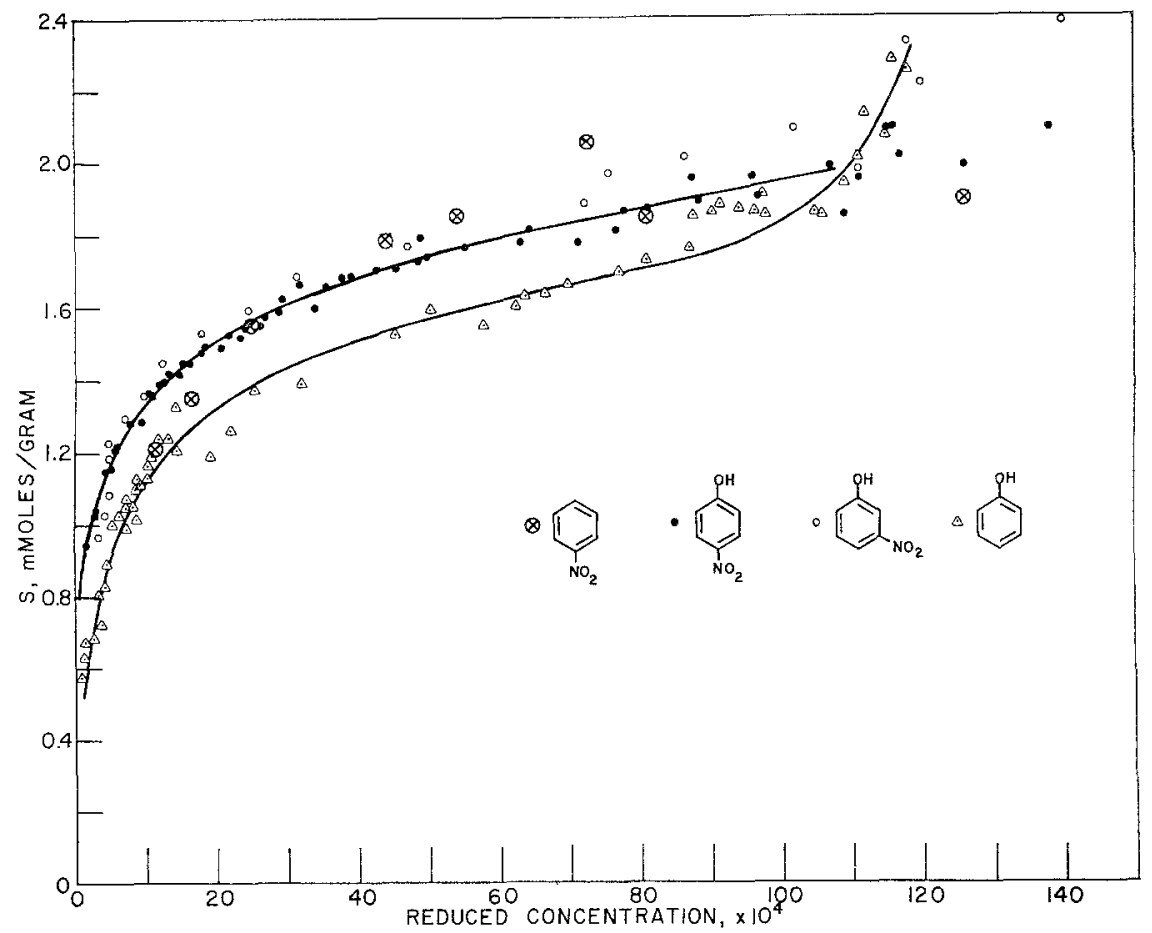

FIG. 3. Reduced concentration isotherms. The isotherms obtained at $25^{\circ} \mathrm{C}$ for phenol, the nitrophenols and nitrobenzene are shown with respect to reduced equilibrium solution concentration, $C_{\text {eq'm }} / C_{\text {s. }}$. 


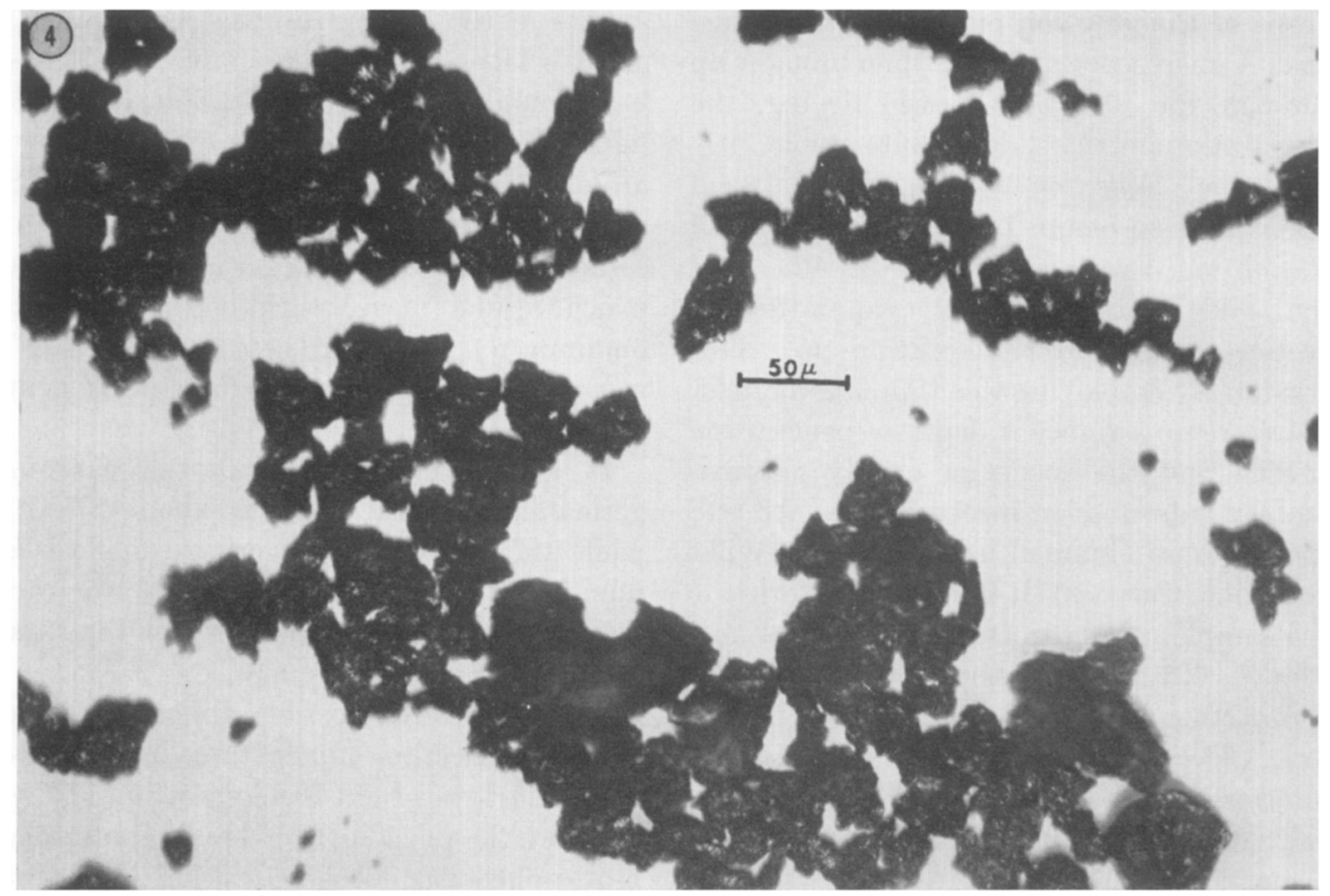

FIG. 4. Nuchar C-1000 activated carbon photomicrograph. An aggregation of the carbon particles used for this study is shown. The particles were sieved through a 325-mesh screen, and the fines removed by washing. The particle size distribution was as follows: ${ }^{2} 0-5 \mu, 14.5 \% ; 5-20 \mu, 22.7 \% ; 20-44 \mu, 62.8 \%$.

IRS crystal is not a true internal reflection spectrum, but instead is a metallic reflection spectrum, where the carbon acts as a metallic reflector (12). The special techniques used to obtain these IRS spectra of carbon are fully described in other publications $(5,12)$. General information on the subject of internal reflection spectroscopy can be found in a comprehensive discussion by Harrick (14).

\section{RESULTS AND DISCUSSION}

Many adsorption processes involving organic molecules result from specific interactions between identifiable structural elements of the sorbate and the sorbent. For purposes of this discussion such interactions are designated as "specific adsorption," as opposed to adsorption which occurs as a result of simple Coulombic interactions. It is possible for specific adsorptive interactions to exhibit a large range of binding energies from the values commonly associated with so called "physical" adsorption, on the lower end of the spectrum to the higher energies involved in so called "chemi"'-sorption. The adsorptive interactions of aromatic hydroxyl and nitro-substituted compounds with active carbon are here considered to be specific adsorption processes.

The adsorption isotherms for phenol, $m$-nitrophenol, $p$-nitrophenol and nitrobenzene on the active carbon are shown in Fig. 3. These isotherms are plotted with respect to the reduced concentration of the sorbate in the bulk solution. ${ }^{8}$ From Fig. 3 it can be seen that all of these species reach the same limiting surface concentration (ca. 2 mmoles/ gram) in the low concentration range (first plateau) covered by the isotherms, which are measured for solute concentrations of less than $1.5 \%$ of the solubility limit. The amount of surface coverage under these conditions

${ }^{8}$ The solubilities are: phenol (pH $2,25^{\circ} \mathrm{C}$ ) $0.47 \mathrm{M} ; m$-nitrophenol (pH $\left.2,25^{\circ} \mathrm{C}\right) 0.045 \mathrm{M}$; $p$-nitrophenol (pH 2, 25ㅇ) $0.042 \mathrm{M}$; nitrobenzene $\left(\mathrm{pH} 2,25^{\circ} \mathrm{C}\right) 0.0118 M$. (16). 


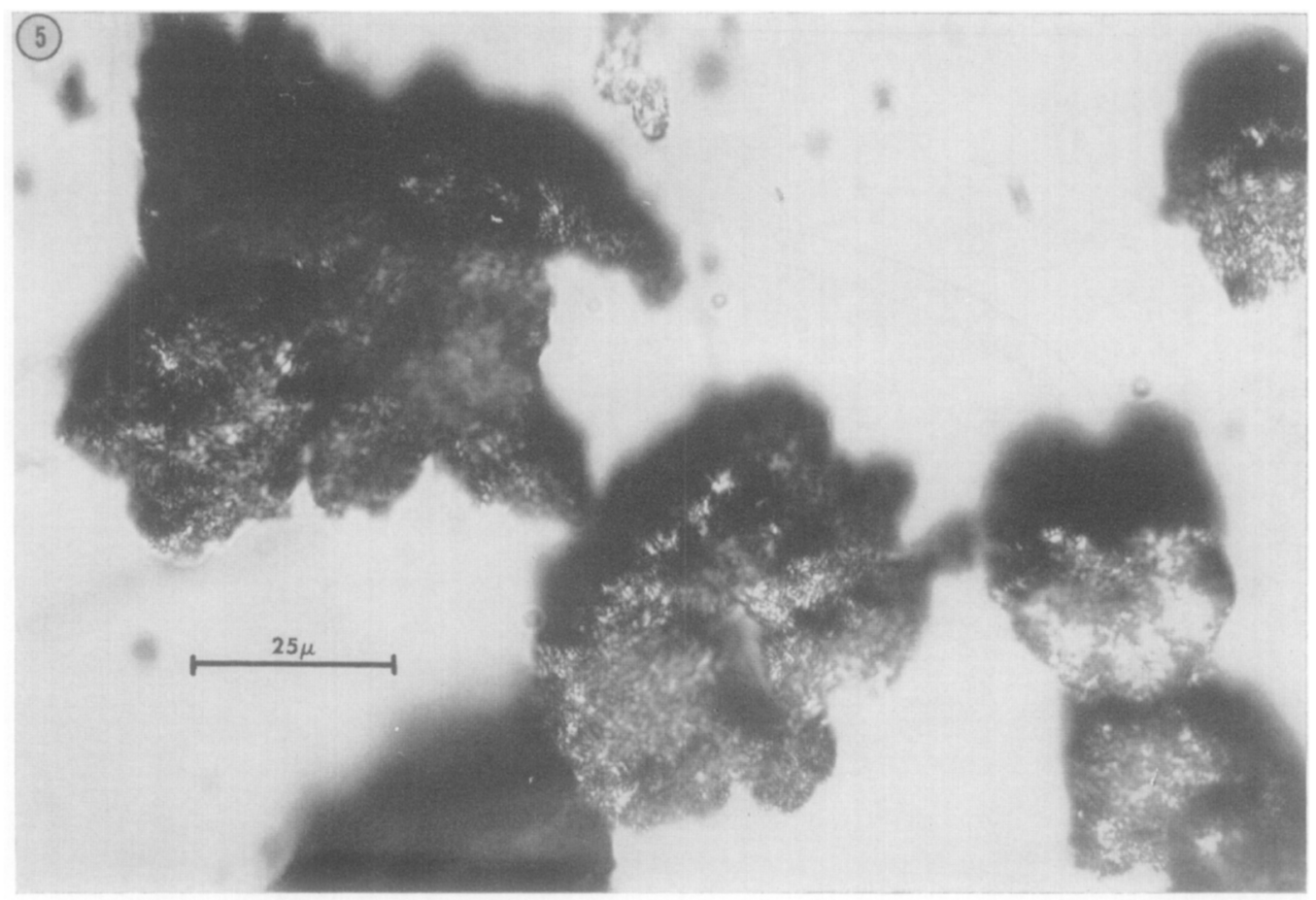

Frg. 5. Nuchar C-1000 photomicrograph. A section of Fig. 4 is magnified about $4 X$ in order to show the highly convoluted surfaces of individual particles.

does not correspond to the total available $\mathrm{N}_{2}$-BET surface area $\left(1000 \mathrm{~m}^{2} / \mathrm{gm}\right)$ available in the carbon used. Assuming an average surface area coverage of about $45 \AA^{2}$ per molecule of an aromatic species in planar orientation $(2,4)$, a surface concentration of $2 \mathrm{mmole} / \mathrm{gm}$ occupies only about $140 \mathrm{~m}^{2} / \mathrm{gm}$, or about $14 \%$ of the total $\mathrm{N}_{2}$-BET surface. This average can be presumed to be in the sub-monolayer region and it may be assumed that the nearest-neighbor interactions on the surface are negligible, although it is possible that the fine pore structure of the carbon used was such as to encourage solute-solute interactions in the form of capillary condensation $(17,18)$. Approximately $40 \%$ of the total pore volume of $0.70 \mathrm{~cm}^{3} / \mathrm{gram}$ was found to be sub-10 $\AA$ radius pores, and about $67 \%$ to be sub-20 $\AA$ pores. ${ }^{\circ}$

A particle size analysis on the carbon used

${ }^{9}$ Andrew Loven, West Virginia Pulp and Paper Co., Covington, Va. (personal communication). in these studies using a dry micro-sieve technique, ${ }^{2}$ showed that the distribution was as follows: $0-5 \mu$ diameter, $14.5 \% ; 5-20 \mu$ diameter, $22.7 \% ; 20-44 \mu$ diameter, $62.8 \%$. Figures 4 and 5 are photomicrographs of the active carbon which show that the particles can be roughly approximated as spheres if a roughness factor is included to account for the irregularities. Using weighted arithmetic average diameters from the particle size distribution, the minimum external surface area for smooth spheres would be about 0.2 $\mathrm{m}^{2} / \mathrm{gm}$. Allowing for a surface roughness estimated from Fig. 5, a roughness factor would give an approximate upper limit for the external surface area of ca. $2 \mathrm{~m}^{2} / \mathrm{gm}$. Approximately $140 \mathrm{~m}^{2} / \mathrm{gm}$ of surface area is occupied by adsorbed solute at the isotherm plateau in Fig. 3, indicating that the majority of adsorbed molecules are on internal surfaces. From the pore size distribution, it would be expected that a large amount of the solute is 


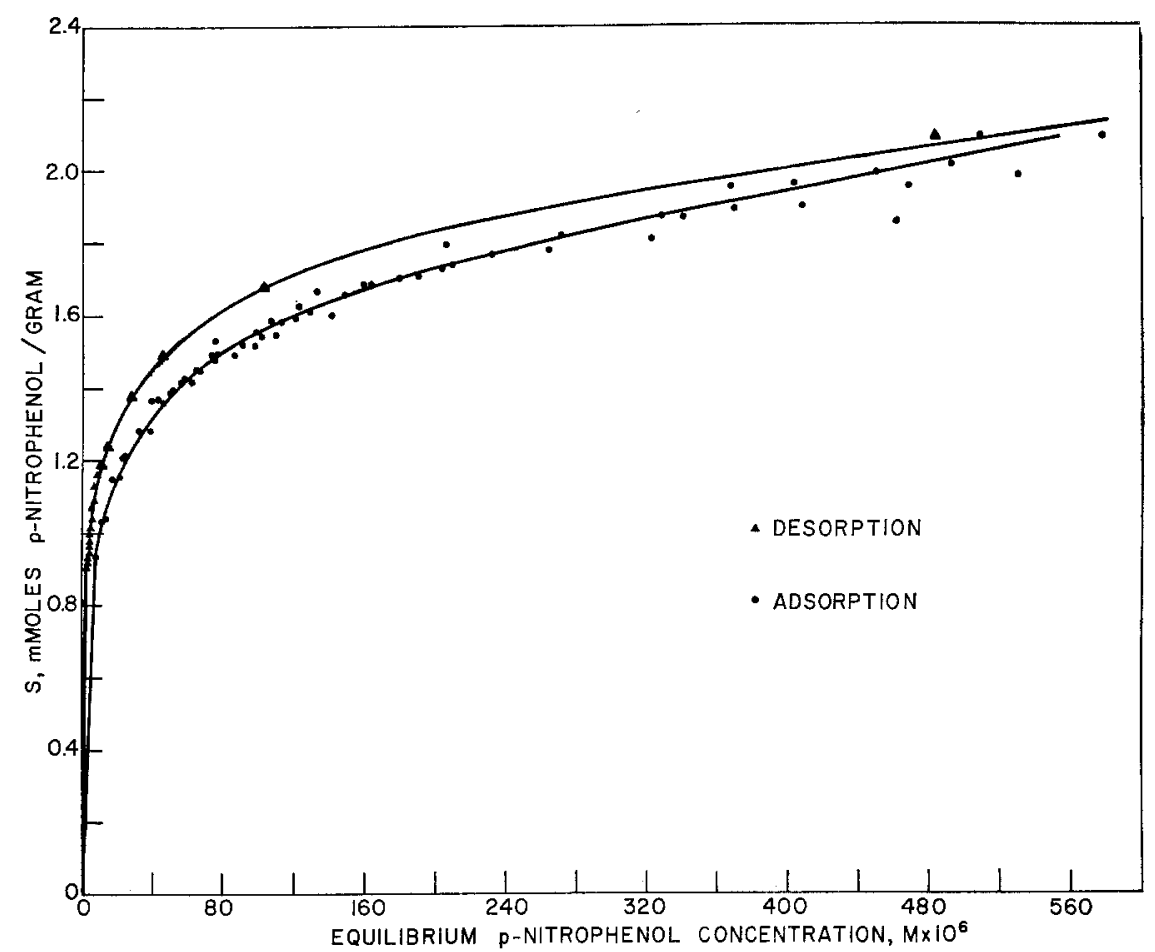

FIG. 6. Adsorption-Desorption of $p$-Nitrophenol. A desorption isotherm for $p$-nitrophenol on the carbon employed in this study is shown along with the adsorption isotherm. Both were obtained at pH 2 and $25^{\circ} \mathrm{C}$.

in very fine pores. In Fig. 6, a desorption isotherm for $p$-nitrophenol as well as the corresponding adsorption isotherm is shown. There is a marked hysteresis effect observed in the desorption curve which indicates that there is an irreversible step in the adsorption-desorption cycle. This behavior suggests the possible occurrence of capillary condensation, even though capillary condensation is usually thought to be a factor only at fairly high concentrations (17). The amount of hysteresis is, however, small and it does not disturb the expected positions of the isotherms under conditions of varying bulk solubility (see Fig. 7). Thus, capillary condensation does not appear to be the major factor in the sorption of phenols on active carbon.

In Fig. 3 the adsorption isotherms for all four solute species are given in terms of their reduced concentrations, and it is important that the relevance and limitations of the use of reduced concentration be understood. Traube's rule can be qualitatively expressed by the relation, $K \cdot C_{\mathrm{s}}=$ Constant, where $K$ is the surface-solution partition coefficient and $C_{\mathrm{s}}$ the solubility. This concept can be used to normalize adsorption isotherms. The use of reduced concentrations, or $C_{\mathrm{eq}} \mathrm{m} / C_{\mathrm{s}}$, normalizes the amount of energy required to bring a molecule of solute from the bulk to the surface of the solution. This normalization is valid only for dilute solutions. Traube's rule can be applied to show that the adsorption of iodine on Graphon is a purely Coulombic interaction, as the temperature dependence of the solubility can be used to account for shifts in the iodine-Graphon adsorption isotherm with temperature $(18, \mathrm{p}$. 102). The extension of Traube's rule to a monofunctional homologous series of organic compounds requires that the interaction, not now restricted to Coulombic effects, be the same in each case, so that only solubility 


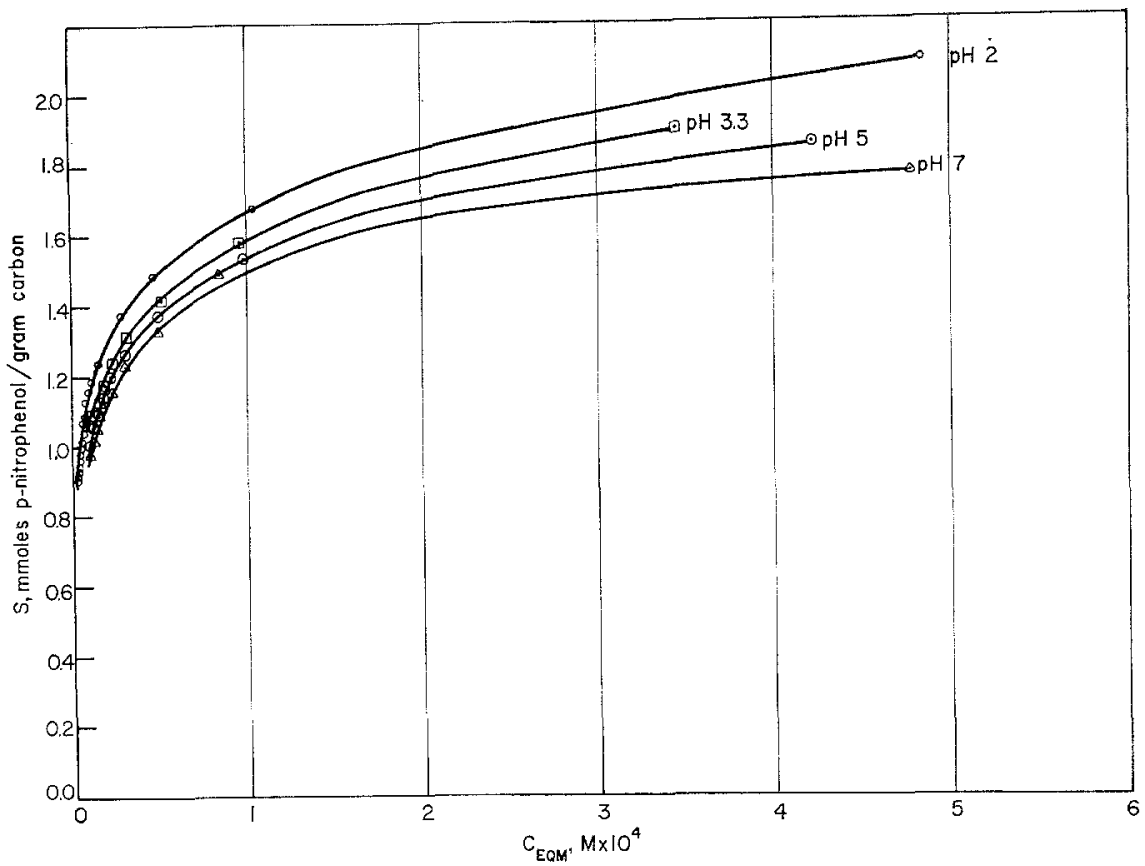

FIG. 7. pH Dependence of $p$-Nitrophenol desorption. Equilibrated samples of $p$-nitrophenol and carbon were subjected to the desorption technique described. Variation of the $\mathrm{pH}$ at which adsorption and deserption were carried out caused only the expected solubility effect.

differences will affect the isotherms. This was shown by Hansen and Craig (17, p. 405) for the adsorption of a homologous series of fatty acids on Spheron 6. By using the reduced concentrations, the adsorption isotherms were found to coincide (for dilute solutions).

In Fig. 3, it can be seen that the reduced concentration isotherms for the nitrophenols both fall very close to one curve, while that for phenol is shifted to the right until it reaches the plateau, and nitrobenzene appears to the right until it reaches the plateau, and nitrobenzene appears to fall in between the two. The near coincidence of the isotherms for $p$ - and $m$-nitrophenol indicates that the interaction for each is the same, regardless of the position of the $\mathrm{NO}_{2}$ group on the ring. The strong adsorption of nitrobenzene indicates a slightly stronger interaction than in the case of phenol, which seems to point to the nitro group as a stronger contributor to the adsorptive interaction than the hydroxyl group.
In Fig. 8, reduced concentration isotherms for $p$-nitrophenol are given at temperatures of $16.3,25.0$, and $40.3^{\circ} \mathrm{C}$. Between 16.3 and $25.0^{\circ} \mathrm{C}$, the solubility only changes from 0.037 to $0.042 \mathrm{M}$, but at $40.3^{\circ} \mathrm{C}$ the solubility is $0.243 M$ (all measurements were made at pH 2). Thus one observes, from Fig. 8, that $p$-nitrophenol adsorption does not follow Traube's with respect to temperature. This implies that the adsorptive mechanism involves more than just coulombic interaction and suggests that some specific interaction is involved.

In many of the $p$-nitrophenol isotherms (see Figs. 8 and 9), there seems to be a break at a surface coverage of about 1.4 mmoles/gram. Snoeyink and Weber (3) have reported a similar break for phenol and $p$-nitrophenol using a different active carbon. There have been previous observations of breaks in the isotherm for the adsorption of phenol on carbon $(2,4)$, but usually these occurred at much higher equilibrium solution concentrations. (This higher concentration 


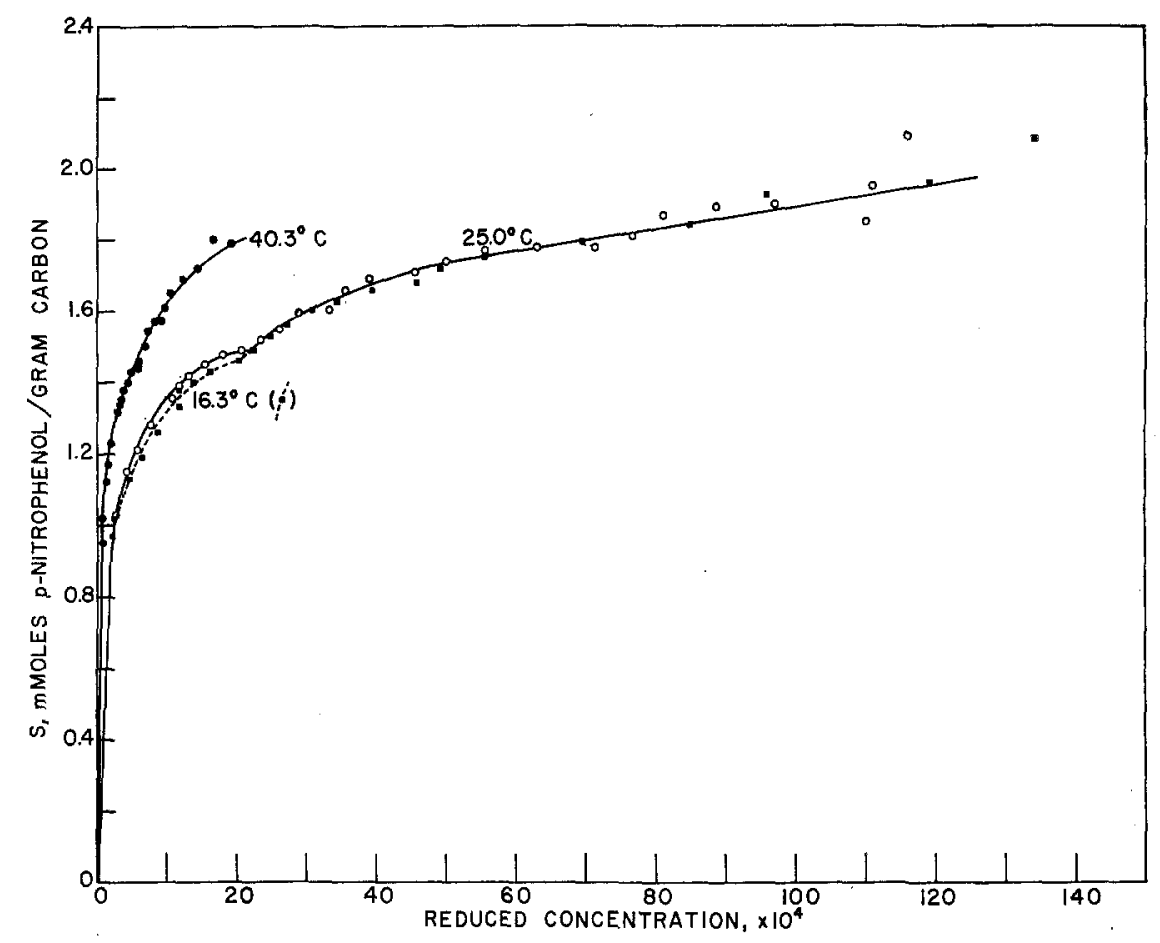

FIG. 8. Reduced concentration isotherms for $p$-nitrophenol at different temperatures.

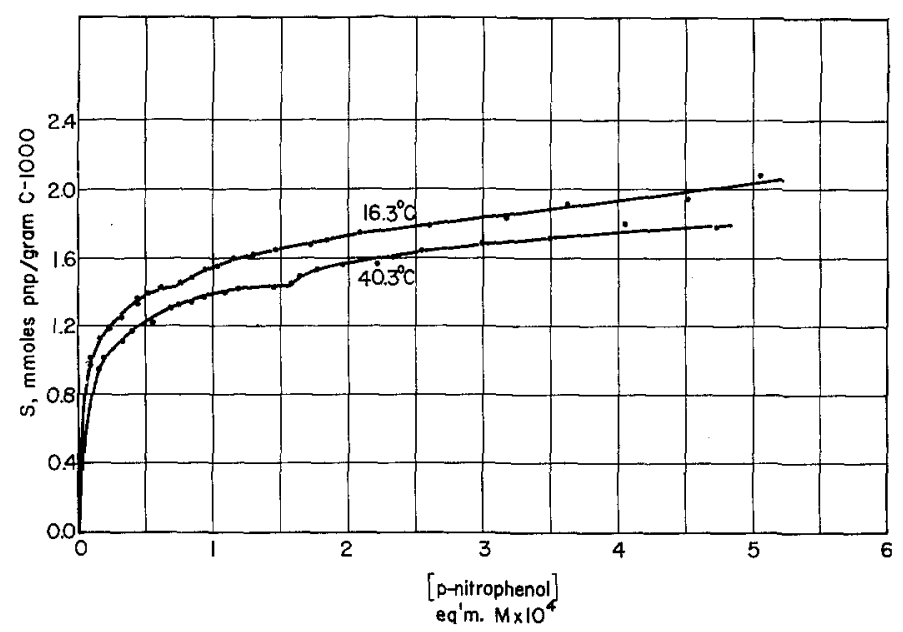

FIG. 9. Isotherms for $p$-nitrophenol at different temperatures.

break in the phenol isotherm is evident in Fig. 3 at the extreme right end of the isotherm). The break at high concentration in the phenol isotherm has been attributed (4) to an uncovering of part of the surface and readsorption in a different orientation. Although the break observed at low equilib- rium solution concentrations is apparently real and of some interest, further study would be required to establish its meaning and significance.

The possible effects of $\mathrm{pH}$ values on these adsorption experiments are three-fold. First, because three of the solutes are weak acids, 
their solubilities are $\mathrm{pH}$ dependent, which results in a shift in the position of the isotherms as a function of $\mathrm{pH}$. This can be seen in Fig. 7, where the effect of $\mathrm{pH}$ on $p$-nitrophenol desorption is shown. Second, the charge on the carbon surface will depend upon the relationship of $\mathrm{pH}$ and the isoelectric point of the carbon. Jones (19) observed that the isoelectric point of a carbon similar to the one used in this study is at $\mathrm{pH}=2.4$. The fact that the desorption experiments in Fig. 7 do not show any significant differences other than solubility effects indicates that the net charge on the carbon surface is not the determining factor in these adsorptions. The third effect is competitive adsorption of $\mathrm{H}^{+}$ions (20). Monitoring of $\mathrm{pH}$ in adsorption and desorption experiments indicated that there was no significant proton concentration change during the course of the experiments (except in the desorption of $p$-nitrophenol at $\mathrm{pH} 7$ ). Thus it can be assumed that competitive adsorption effects were held constant throughout the experimental work by using $\mathrm{HCl}$ solution at $\mathrm{pH}$ 2 for all studies. It is important, of course, that $\mathrm{pH}$ be held constant in the adsorption studies of weak acids to avoid solubility effects on the isotherm which would unnecessarily complicate the interpretation of isotherm data. ${ }^{10}$

In order to observe the structural changes undergone by these compounds after adsorption on active carbon, infrared spectra of the surfaces after adsorption were $o b$ tained by an internal reflection technique described in earlier work $(5,12)$. The IRS spectrum of solid $p$-nitrophenol is shown in Fig. 10. The observed bands are identified as follows: The broad $3100-3500 \mathrm{~cm}^{-1}$ band is due to the $\mathrm{O}-\mathrm{H}$ stretch of strongly intermolecularly hydrogen bonded $\mathrm{O}-\mathrm{H}$; the bands at 1611,1590 , and $1506 \mathrm{~cm}^{-1}$ are aromatic $\mathrm{C}-\mathrm{C}$ stretching vibrations; the bands at 1497 and $1284 \mathrm{~cm}^{-1}$ are $\mathrm{NO}_{2}$ bands;

${ }^{10} \mathrm{R}$. W. Coughlin, Department of Chemical Engineering, Lehigh Univ., Bethlehem, Pa. (personal communication). the strong band at $1330 \mathrm{~cm}^{-1}$ is due to the phenolic $\mathrm{C}-\mathrm{O}$ stretch; the band at $1214 \mathrm{~cm}^{-1}$ is the $\mathrm{O}-\mathrm{H}$ bending vibration; the bands at 1165 and $1110 \mathrm{~cm}^{-1}$ are aromatic ring vibrations; and the triplet around $850 \mathrm{~cm}^{-1}$ is due to ring $\mathrm{C}-\mathrm{H}$ vibrations. The broad absorption around $1300-1100 \mathrm{~cm}^{-1}$ and the shoulder at $1200 \mathrm{~cm}^{-1}$ are due to a Teflon O-ring holding the crystal in place.

Spectra obtained by the IRS technique developed for carbon surfaces cover the entire group frequency region of the infrared spectrum (2.5-15 $\mu$ with Ge; $2.5-40 \mu$ with KRS-5), and can be used to infer how the organic solute molecules interact with the carbon in the adsorbed state. General experimental problems associated with this technique are presented in earlier papers $(5$, 12 ), and will not be discussed here. With the carbon used in this study, the external surface area was approximately $1-2 \mathrm{~m}^{2} / \mathrm{gm}$, and the average surface coverage was only about $14 \%$ of all available $\mathrm{N}_{2}$-BET surface area, so that it was necessary to push the internal reflection technique to the limits of its sensitivity in order to obtain good spectra $(5,12)$. The photomicrograph in Fig. 5 shows that the particles had highly convoluted surfaces, and it is not possible to obtain good optical contact between these particles and the IRS crystal (12).

Figure 2 shows several IRS spectra taken in the $1600-1200 \mathrm{~cm}^{-1}$ region. The uppermost of these spectra is reproduced from the pure $p$-nitrophenol spectrum in Fig. 10. The lower right spectrum, from 1400 to 1280 $\mathrm{cm}^{-1}$, was taken on a sample of carbon after the adsorption of $p$-nitrophenol. ${ }^{\text {II }}$ This spectrum clearly shows that the phenolic $\mathrm{C}-\mathrm{O}$

1 The sample was equilibrated at $S=2.0$ mmoles $p$-nitrophenol/gram, and subsequently desorbed to $S=1.6 \mathrm{mmoles} / \mathrm{gm}$, then washed and dried to assure that no $p$-nitrophenol was left on the surface from evaporation of interstitial solution. The procedure was checked by reimmersing a portion of the dried sample in the last equilibrium solution and measuring the uptake of solute. The losses were calculated to be less than $6 \%$ of the equilibrium $S$ value. 


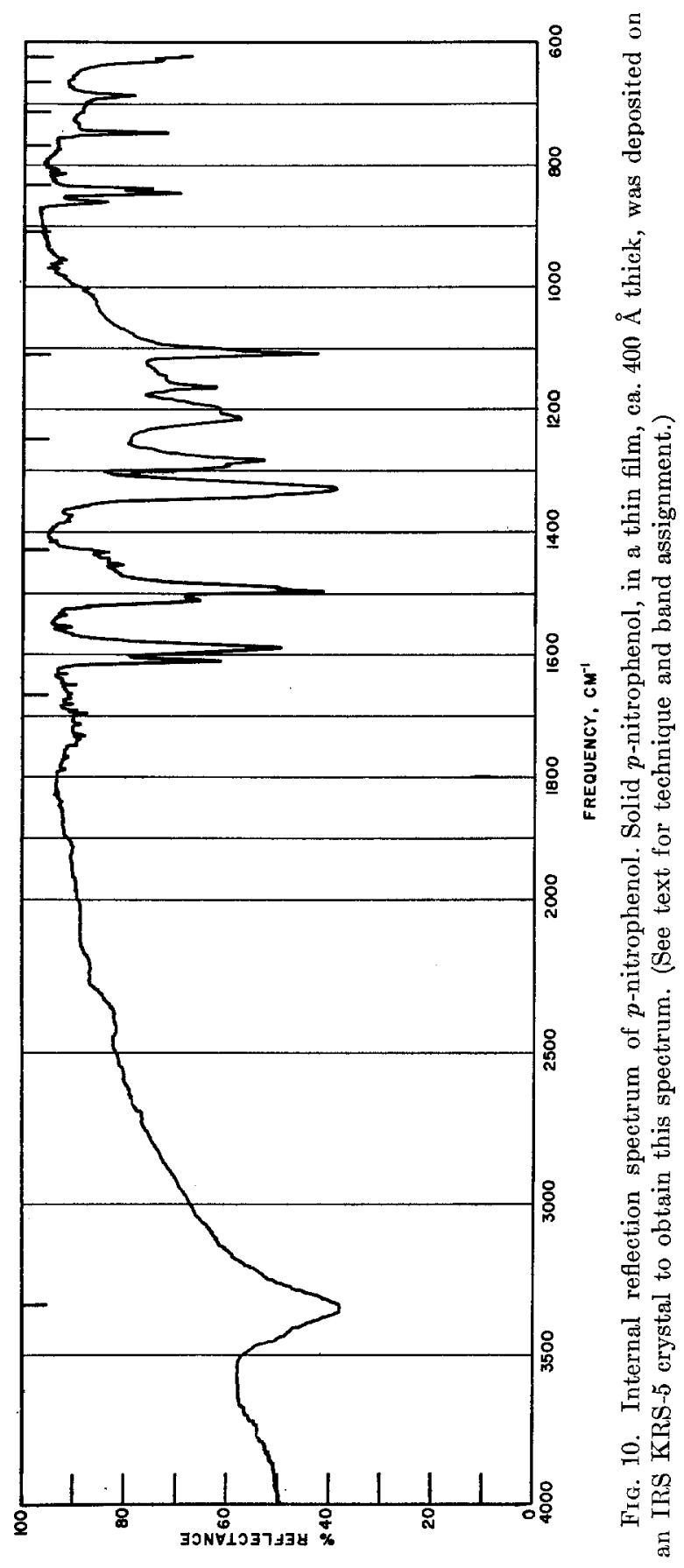


vibration is in the same position, and has the same shape as in the pure p-nitrophenol spectrum. This spectrum was obtained using $20 \times$ scale expansion of a 6 reflection spectrum of carbon on KRS-5 (60 $)$. The adjacent spectrum in the lower left part of Fig. 2 was taken with the same IRS geometry, but is a $10 \times$ scale expansion. This spectrum, which has been corrected for partial interference of water vapor in the beam, shows that the nitro group peaks are essentially unchanged with respect to their positions before adsorption.

Figure 1 reproduces the intermolecularly hydrogen bonded $\mathrm{O}-\mathrm{H}$ band $(\mathrm{O}-\mathrm{H} \cdots \mathrm{O})$, and below this spectrum are (i) a $60^{\circ} \mathrm{KRS}-5$ $10 \times$ baseline, and (ii) the spectrum of the carbon with adsorbed $p$-nitrophenol. Both (i) and (ii) were taken at $10 \times$ scale expansion. It is clear from Figure 1 that the strong $O-H \cdots O$ band has disappeared upon adsorption.

From these spectral data, some definite conclusions regarding the surface structure of $p$-nitrophenol can be drawn. First, since the phenolic $\mathrm{C}-\mathrm{O}$ band is unchanged after adsorption, the oxygen cannot be directly associated with the surface in any specific interaction, since any such interaction would be expected to alter the $1330 \mathrm{~cm}^{-1}$ band observed in Fig. 2. Second, it is evident that the phenolic hydrogen is either gone, or is in an energetically symmetric environment which significantly reduces its infrared activity by virtue of symmetry. Loss of this hydrogen does not agree with what is observed regarding the $\mathrm{C}-\mathrm{O}$ bond. Such a loss would cause the $p$-nitrophenol to revert to its most stable anionic structure, which is a quinoid structure. This would give the $\mathrm{C}-\mathrm{O}$ bond considerable double bond character, and shift the peak up into the $1600-1660 \mathrm{~cm}^{-1}$ region. No such new bands were observed in the spectra taken in this region, nor were any $\mathrm{C}=\mathrm{C}$ bands of a quinoid structure observed. Thus it is most likely that the hydrogen is involved in intracomplex hydrogen bonding, within the surface complex itself. It has been shown in Gould (20) that the O-H stretching vibra- tion in such a case will considerably diminish, broaden, and possibly even disappear altogether.

\section{CONCLUSIONS}

The reversibility of the adsorption (Fig. 6) and the amount of temperature dependence not corrected for by solubility effects (Fig. 8 ) indicate that a weak chemical interaction probably accounts for the adsorption of these compounds. In this respect, the adsorption characteristics of the various solutes are examined on the basis of their structures and their reactivities in homogeneous solution. This information is correlated with the spectral data in an attempt to determine the most likely mechanism which is consistent with all that is known about the systems. The structure of the solutes can be divided into three reactive functionalities, (i) the $\mathrm{O}-\mathrm{H}$ group, (ii) the $-\mathrm{NO}_{2}$ group, (iii) and the aromatic ring pi-electron system.

The surface of activated carbon has been described $(1-3,5-12)$ as a collection of organic functional groups containing oxygen, with these groups occurring primarily at the edges of broken graphitic planes, and basal planes consisting of large fused aromatic ring systems in a graphite-like structure. $\mathrm{Hy}$ drogen bonding by the phenolic protons with these oxygen groups is one possible surface interaction in light of the typical interactions observed in solution. This would not account, however, for the fact that nitrobenzene, which has no such proton, is adsorbed as strongly as the phenolic molecules (Fig. 3). If this mechanism were of primary importance, it would be expected that the isotherms of these weak acids would correlate on the basis of their relative $p K_{\mathrm{a}}$ values. Thus major differences would be expected between $p$-nitrophenol $\left(p K_{\mathrm{a}}=7.15\right), m$-nitrophenol $\left(p K_{\mathrm{a}}=8.2\right)$ and phenol $\left(p K_{\mathrm{a}}=9.8\right)$. Such differences are not observed (Fig. 3). Any interaction based on the electron donating ability of the phenolic oxygen would be discounted by the same $p K_{\mathrm{a}}$ argument, as well as the spectral evidence for the non-involve- 
ment of the oxygen. On the basis of the disappearance of the $\mathrm{O}-\mathrm{H}$ stretch in Fig. 1, as well as the position of the nitrobenzene isotherm between phenol and nitrophenol, it is evident that there is some hydrogen bonding with surface groups involved, but this interaction is small and is not the primary cause for adsorption of phenols.

If the nitro group were primarily responsible for the adsorption of the nitrophenols and nitrobenzene, one would not expect the strong adsorption observed for phenol (Fig. 3). Also, the spectral evidence in Fig. 2 shows that the nitro group does not undergo significant changes upon adsorption. Thus, one must conclude that the nitro group is not directly involved in the solutecarbon interaction.

The interaction of the aromatic ring with the surface of the active carbon must, therefore, be considered the major influence in these processes, interacting through the pielectron system of the ring. There is considerable evidence in the literature for the formation of donor-acceptor ${ }^{12}$ complexes between phenol and several kinds of electron donors (2). One can say that two factors will influence the stability of such complexes; (i) the availability of electron density in the donor, and (ii) the electron affinity of the acceptor.

It is well known $(21,22)$ that the electron density of an aromatic ring is strongly influenced by the nature of the substituent groups. A nitro group acts as a strong electron withdrawing group in reducing the overall electron density in the pi-system of the ring. Thus, nitro-substituted aromatic compounds act as acceptors in such com-

12 A donor-acceptor complex is a complex resulting from a small amount of orbital mixing between an electron-rich and an electron-deficient species. The binding energies of donor-acceptor complexes can range from 2 to $15 \mathrm{kcal} / \mathrm{mole}(21)$, and is partially covalent (less than 10\%) and partially Coulombic in nature. Although donoracceptor complex is the accepted term, frequently the same phenomenon is described as a chargetransfer complex (22). plexes and form stronger donor-acceptor complexes with a given donor than phenol, because the phenol has no low-lying acceptor orbitals (22) and forms complexes with very strong donors. The isotherms for $m$-nitrophenol and $p$-nitrophenol are essentially identical, indicating that the net energy of interaction at the surface is comparable for both (Fig. 3).

Drago (22) has shown that phenol forms strong donor-acceptor complexes with oxygen groups, and it is known that the oxygen group dipole moment is the determining factor in the strength of the donor-acceptor complex formed. Carbonyl oxygen has a larger dipole moment than carboxylic acid oxygen, and thus would be expected to act as the stronger donor $(21,22)$. Thus, it is suggested that these aromatic compounds adsorb on active carbon by a donor-acceptor complex mechanism involving carbonyl oxygens of the carbon surface acting as the electron donor and the aromatic ring of the solute acting as the acceptor. These complexes would be expected to behave reversibly; and because of the pi-system interaction, it is expected that the solute molecules would adsorb in the planar orientation.

As mentioned in the introduction, Coughlin and Ezra (2) observed that a change in the adsorptive capacity for phenol and nitrobenzene took place upon oxidation, or reduction, or both of the carbon surface. They (2) observed that upon oxidation of the surface, both capacities decreased, and with reduction, both capacities increased. Boehm (11) and Coughlin and Ezra (2) show that the oxidation of the carbon surface increases the amount of strongly acidic oxygen-containing functional groups through the oxidation of carbonyl groups. They interpret the reduction as being the reverse of the above process. As it cannot be expected that the $\mathrm{C}-\mathrm{C}$ bonds broken in oxidation will rejoin upon reduction, much of the original carbonyl groups are not reformed, hence the capacity may not fully recover to its original value. Thus, it is reasonable to expect that 
the capacity of carbon for electron acceptors would go down upon oxidation of surface carbonyl groups to carboxylic acid groups. It is interesting to note that the data of Coughlin and Ezra (2) also seem to indicate that the second phenol plateau is not affected by oxidation of the surface, which would lead one to expect that the second step in phenol adsorption is the formation of a donor-acceptor complex with the fused ring system of the basal planes of the carbon.

All of the data support the mechanism of a donor-acceptor complex with surface carbonyl oxygen groups, with adsorption continuing after these sites are exhausted by complexation with the rings of the basal planes. It should also be mentioned that the relative rates of adsorption of the various compounds studied were measured in order to determine the time necessary for equilibration of isotherm samples. These relative rates are consistent with the proposed mechanism, since the nitro-substituted compounds would be expected to react faster from a linear free energy argument (22). If the proposed mechanism is correct, then it should be possible to predict the behavior of di- and trinitrophenol as well. DiGiano and Weber (13), in recent adsorption studies, examined the relative amounts of adsorption of 2,4-dinitrophenol and $p$-nitrophenol on the same active carbon, and found that the dinitrophenol isotherm was shifted considerably to the left of the $p$-nitrophenol isotherm, indicating a much stronger interaction for dinitrophenol. This is exactly what is predicted by the proposed mechanism. Although no data is available it would be expected that trinitrophenol will adsorb even more strongly on active carbon than any of the species mentioned here. Known trinitrophenol charge transfer complexes (picrates) are often so stable that they can be isolated and crystallized from solution.

\section{ACKNOWLEDGMENTS}

The authors wish to acknowledge Wilks Scientific Corp., S. Norwalk, Conn., for the donation of internal reflection equipment, and Paul Wilks for his help and advice in the selection of IRS crystals and techniques. The University of Toledo Department of Chemistry is gratefully acknowledged for allowing the authors to use their Perkin-Elmer 621 spectrophotometer, and the West Virginia Pulp and Paper Company is acknowledged for their cooperation in obtaining carbon samples, as well as pore size, particle size and BET surface area information.

This research was supported in part by the Western Electroanalytical Theoretical Society and the Federal Water Pollution Control Administration Grant No. WP-0076, and the National Science Foundation, Grant No. GP-9307.

Presented at the 43rd National Colloid Symposium, Colloid and Surface Chemistry Division, ACS, Case Western Reserve University, Cleveland, Ohio, June 23-25, 1969.

\section{REFERENCES}

1. Snotyink, V. L. and Weber, W. J., JR., Environ. Sci. Technol. 1, 228 (1967).

2. Coughtis, R. W. ANd EzRA, F. S., Environ. Sci. Technol. 2, 291 (1968).

3. Snoeyink, V. I., and WeBer, W. J., JR., Technical Publication T-68-3, Dept. of Civil Engineering, University of Michigan (1968).

4. Giles, C. H., MacEwan, T. H., NakHwa, S. N., and SMith, D., J. Chem. Soc. 1960, 3973.

5. Mattson, J. S., Mark, H. B., JR., AND Weber, W. J., JR., Anal. Chem. 41, 355 (1969).

6. Garten, V. A. And Weiss, D. E., Australian J. Chem. 8, 68 (1955).

7. - - A ust. J. Chem. 10, 309 (1957).

8. Garten, V. A., Weiss, D. E., AND Willis, J. B., Australian J. Chem. 10, 295 (1957).

9. Garten, V. A. and Weiss, D. E., Rev. Pure Appl. Chem. 1, 69 (1957).

10. Garten, V. A. And Eppinger, K., Australian J. Chem. 12, 394 (1959).

11. Boenм, H. P., Advan. Catalysis 16, 179 (1964).

12. Mattson, J. S. and MARK, H. B., JR., $J$. Colloid and Interface Sci., 31, 131 (1969).

13. DiGrano, F. J., and WEBER, W. J., JR., Technical Publication T-69-1, Dept. of Civil Engineering, University of Michigan, (1969).

14. Harrick, N. J., "Internal Reflection Spectroscopy," Interscience New York (1967).

15. Foster, P. J. and Howarth, C. R., Carbon 6, 719 (1968).

16. "Solubilities of Inorganic and Organic Compounds," 1308-1310, Vol. 1. (H. Stephan and T. Stephan, Eds.) MacMillan, New York (1963).

17. Adamson, A. W., "Physical Chemistry of 
Surfaces," 2nd Ed., Interscience New York (1967).

18. KrPLING, J. J., "Adsorption From Solutions of Non-electrolytes," Academic Press, London (1965).

19. Jones, C. R., M. S. Thesis, University of Maryland (1968).

20. SNOEYINK, V. L. and WeBer, W. J., JR., "Adsorption from Aqueous Solution," Advances in Chemisty Series, 79, ed. by R. F. Gould, ACS Publications, Washington, D.C., 1968, pp. 112-134

21. Gould, E. S. "Mechanism and Structure in Organic Chemistry," p. 30, Holt, Rinehart and Winston, New York (1959).

22. Drago, R. S., Niedzielski, R. J., Middaugh R. L., J. Am. Chem. Soc. 86, 1694 (1964).

23. Tamres, M. R., J. Chem. Ed. 45, 221 (1968). 